\title{
Probing early parton kinetics by photons, dileptons and charm*
}

\author{
B. Kämpfer $^{\mathrm{ab}}$ and O.P. Pavlenko ${ }^{\mathrm{c}}$
}

${ }^{a}$ Research Center Rossendorf, PF 510119, 01314 Dresden, Germany

bInst. Theor. Phys. (KAI e.V.), Technical University Dresden, Germany

'Inst. Theor. Phys. Kiev, Ukraine

Equilibration processes in pre-equilibrium parton matter are considered. We investigate chemical quark equilibration, partial thermalization and overall thermalization, and their influence on electromagnetic (photons, dileptons) and charmed probes.

\section{Introduction}

The future heavy-ion colliders RHIC and LHC will achieve energy regions where many semihard-scattered, secondary partons are produced. The estimated energy densities are so large that the dense parton matter is expected to stay for some time in a deconfined state. Whether the partonic interactions are efficient enough to bring the system in a quark-gluon plasma state is still matter of debate. Also the identification of the transient plasma, whence created, is a difficult task, despite of many proposed signals.

Nowadays, parton cascade models [1-3] are under development, which are aimed to follow the parton evolution in the course of a nuclear collision on grounds of improved perturbative QCD. Since such cumbersome codes need some numeric efforts, it seems to be desirable to model various evolution aspects by more transparent analytical simulations.

It is the aim of the present contribution to describe equilibration processes in preequilibrium parton matter in a sufficiently tracktable way to get some insight in their importance for plasma probes. In the last decade most probes are calculated for a fully locally equilibrated quark-gluon plasma [4]. Since the initial parton distribution in two separated nuclei differs totally from such a plasma state, there must be a pre-equilibrium stage before the plasma era, and its relevance for the probes has been till recently a miracle. Here we go a step into past and consider the pre-equilibrium prior to the plasma. This does not resolve the entire history from the very beginning of a nuclear collision, but might demonstrate how important the pre-equilibrium parton matter is.

We consider three scenarios:

- (i) Due to the relations of total cross sections $\sigma_{g g}>\sigma_{g g}>\sigma_{q q}$, a dense and hot, thermalized gluon plasma should be created first, according to Ref. [5] at proper time $\tau \approx$

*supported by BMFT under grant $06 \mathrm{DR}$ 107;

invited talk at Quark Matter '93 (Borlänge), to be published in Nucl. Phys. A, Proceedings of the 10th International Conference on Ultrarelativistic Nucleus-Nucleus Collisions 
$0.5 \mathrm{fm} / \mathrm{c}$. The quark admixture is rather low, according to Refs. $[1,3,5,6] 30 \%$ of its chemical equilibrium value. Relying on a simple transport model of Boltzmann type we calculate within lowest order $\alpha_{s}$ processes the quark chernical equilibration process. As application we consider photon production in such an initially gluon-enriched plasma.

- (ii) In line with the above quoted cross sections the glue system thermalizes earlier than quarks. Assuming primordial secondary-parton production in minijets, the quark component might possess some time another distribution than gluons till complete thermalization. Since the minijet picture predicts a power-law high $p_{\perp}$-tail, such tails might be favorable for producing large invariant mass probes such as charm pairs.

- (iii) At very early times all partons are expected to be off-equilibrium. Within a simplified kinetic model we follow the evolution towards thermal equilibrium. As usefull frame-independent probe we consider dileptons.

\section{Chemical equilibration of quarks/photons}

\subsection{Quark cooking}

The boost invariant Boltzmann equation

$\left(\partial_{\tau}-\frac{\operatorname{th} \xi}{\tau} \partial_{\xi}\right) f_{a}=E_{a}^{-1} \mathcal{C}_{a}$

describes the evolution of parton species $a=g, q, \bar{q}$ with energy $E_{a}$ in terms of their distribution functions $f_{a}$ due to collisions $(\mathcal{C}=$ collision term) as function of the proper time $\tau=\sqrt{t^{2}-z^{2}}$ and rapidity $\xi(\xi=\eta-y, \eta / y=$ space-time/parton rapidity). Phase space integration gives the evolution equation of densities $\left(n=\int d \Gamma f, d \Gamma \equiv g(2 \pi)^{-3} d^{3} p\right)$

$\frac{d n_{a}}{d \tau}+\frac{n_{a}}{\tau}=\int d \Gamma_{a} \mathcal{C}_{a}\left(p_{a}\right)$

Using, according to the above outlined evolution scenario (i), thermal distributions $f_{e q}$ in Maxwell Boltzmann approximation, one gets

$\frac{d n}{d \tau}-\frac{n}{\tau}=0, \quad \frac{d x}{d \tau}=n \mathcal{R}\left(\beta[1-x]^{2}-x^{2}\right)$,

where $n=n_{g}+n_{q}+n_{\bar{q}}$ and $x=n / n_{g}$ stand for total parton density and gluon weight, and $\beta=4 / 9$. In case of lowest order $g g \rightleftharpoons q \bar{q}$ reactions the reactivity $\mathcal{R}$ becomes

$\mathcal{R}=\frac{\pi \alpha_{s}^{2}}{24 m^{2}}\left(\frac{m}{T}\right)^{5} \mathcal{I}(m, T), \quad \mathcal{I}=\int_{2}^{\infty} d Z Z^{2} \sigma(Z) K_{1}\left(\frac{m Z}{T}\right)$

$\sigma(Z)=\left(1+4 Z^{-2}+Z^{-4}\right) \operatorname{arth} W-\left(7 Z+31 Z^{-2}\right) \frac{W}{8}, \quad W \equiv \sqrt{1-4 Z^{-2}}$

with regularizing thermal quark mass $m^{2}=T^{2} 2 \pi \alpha_{s} / 3$. The evolution of the relative quark weight proceeds as dispayed in Fig. 1 . In accord with other estimates $[3,6,8]$ one observes a rather slow cooking of quarks. The longitudinal expansion hinders oviously the quark chemical equilibration. Processes $g g \rightarrow g g g[6]$ prevent the fast gluon dilution 


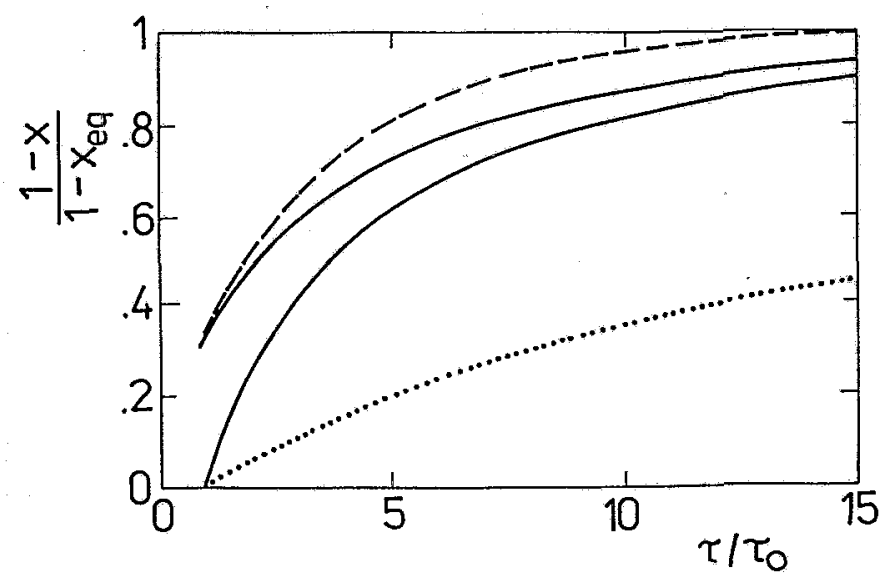

Figure 1. Time evolution of the relative quark weight for different initial values. Full and dashed lines use $x_{0}=0.8, n_{0}=10 \mathrm{fm}^{-3}, T_{0}=500 \mathrm{MeV}$, $\tau_{0}=0.5 \mathrm{fm} / \mathrm{c}, \alpha_{s}=0.58$, while the dotted line depicts an example with a three times smaller value of $n_{0} \tau_{0} T_{0}^{-2}$; dashed line: without expansion. For details see Ref. [7].

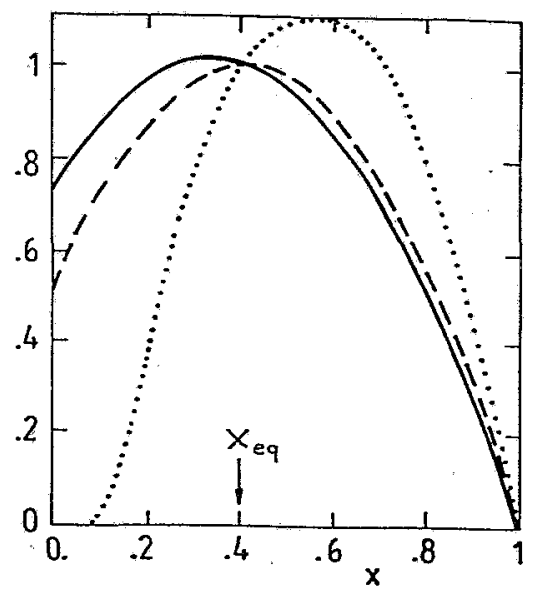

Figure 2. The photon yield per fourvolume in units of the equilibrium yield $\left(x_{e q}=0.4\right)$ for different photon energies (full/dashed/dotted lines $E=3 / 2 / 1 \mathrm{GeV}$ ). In both yields the same total parton density is assumed.

(mimicked here by switching off the expansion), and higher order processes $g g \rightarrow n g$, $n \geq 4$ further damp the gluon dilution [9]. But the dashed line in Fig. 1, which is without expansion, indicates anyway a slow equilibration. The inelastic processes also change the temperature evolution, which proceeds for $2 \rightleftharpoons 2$ reactions according to eq. (1) as $T=T_{0}\left(\tau_{0} / \tau\right)^{1 / 3}$.

\subsection{Photon yield}

The photon yield according to Ref. [10] is for $q \bar{q} \rightarrow g \gamma$ and $q g \rightarrow q \gamma$ processes

$\frac{d N_{\gamma}}{d^{4} x d^{3} p / E}=\frac{5 \alpha \alpha_{s} n^{2}}{72 T^{4}} \mathrm{e}^{-E / T}\left\{\left[\log \frac{3 E}{\pi \alpha_{s} T}-C_{E}\right]\left(2-x-x^{2}\right)-\frac{1}{2}\left(4-11 x+7 x^{2}\right)\right\}$

$\left(C_{E}=\right.$ Euler's constant) with an infrared cut-off $k_{c}^{2}=T^{2} 4 \pi \alpha_{s} / 3$. As seen in Fig. 2 the yield is strongly suppressed for the gluon enriched plasma, i.e., at $x \rightarrow 1$. There are indications from the HIJING simulations [6] that in early stages the parton density is diminished, compared to full equilibrium density at given temperature. This would cause an additional suppression. The parton cascade [1] does not show such a pronounced parton suppression; this might be due to different low-momentum cut-off's.

The time integrated yield is displayed in Fig. 3 in relative units. As seen in the lower part the main affect on the yield comes from the initial temperature. Compared with these large variations the suppression of the photon yield due to reduced quark concentration is a minor effect as long as the initial quark contribution is not smaller than $30 \%$ of its chemical equilibrium value. A sufficiently fast chemical equilibration affects the slopes, 


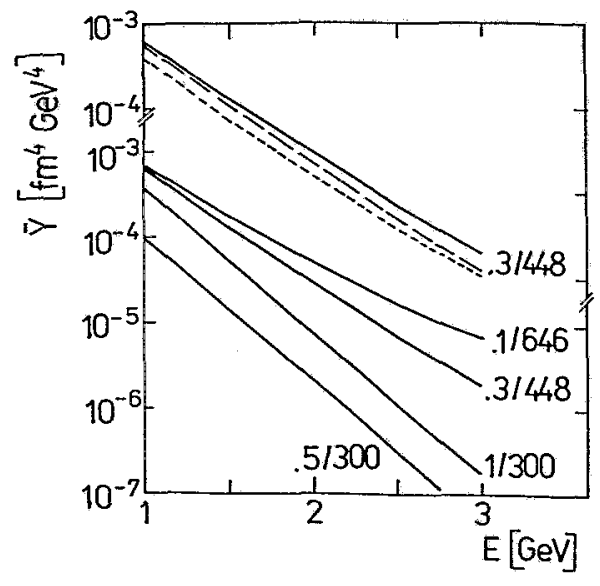

Figure 3. Time integrated photon yield $\bar{Y}=\frac{d N_{\gamma}}{d \eta d^{3} p / E} / \pi R^{2} \frac{5 \alpha \alpha_{s} n_{0}^{2} \tau_{0}^{2}}{72^{2} T_{0}^{6}}$ as function of energy. Lower part: equilibrium yields for different initial times (in $\mathrm{fm} / \mathrm{c}$ ) and temperatures (in $\mathrm{MeV}$ ), $\tau_{0} / T_{0}$. Upper part: different evolutions of the quark weight (full/dashed [long-dashed] lines $x=x_{e q} / x_{0}=0.8$ and slow [fast] equilibration).

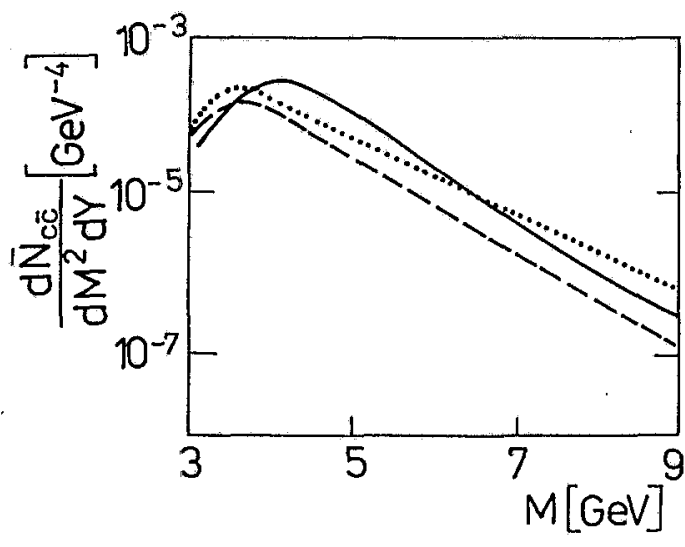

Figure 4. Number of charmed pairs $d \bar{N}_{c \bar{c}} / d M^{2} d Y\left(N_{c \bar{c}}=\bar{N}_{c \bar{c}} \pi\left(R \alpha_{s} n_{0} \tau_{0}\right)^{2}\right)$ as function of invariant mass. Full (dashed) line: pairs from power-law (thermal) quarks, dotted line: yield from thermal gluons. The evolution is terminated when reaching an energy density corresponding to $T_{f}=200 \mathrm{MeV} . \tau_{0}=1 / p_{\perp}^{*}$ is used.

which might be used to measure the degree of quark equilibration. The absolute yields might serve as thermometer. As Ref. [3] we conclude that a higher initial temperature in the hot glue scenario more than overcompensates the lack of quarks.

Note however that the photon energy is frame dependent. A substantial transverse expansion would enhance the large- $E$ yield [11]. For a comparison of the parton yield with later hadronic sources see Refs. $[3,10,11]$.

\section{Partial thermalization/charm}

Minijets are known to obey a power-law $p_{\perp}$ distribution [12]. Due to the correlation of momentum space and configuration space, the very early parton distribution in a localized volume element might be parametrized as

$f_{0}\left(p_{\perp}, y, \tau_{0}\right)=\mathcal{N} \Theta\left(p_{\perp}-p_{\perp}^{*}\right) \delta(y) p_{\perp}^{-l}, \quad l \approx 7$

with $p_{\perp}^{*}$ as regularizing low-momentum cut-off and $\mathcal{N}$ as normalization. As shown in Refs. $[13,14]$ an initial distribution $f_{0}$ evolves, according to the relaxation time approximation to eq. (1), as

$$
f(\tau)=f_{0}(\tau) \exp \left\{\frac{\tau_{0}-\tau}{\tau_{\text {rel }}}\right\}+\mathcal{F}, \quad \mathcal{F} \approx\left(1-\exp \left\{\frac{\tau_{0}-\tau}{\tau_{\text {rel }}}\right\}\right) f_{\text {eq }} \text { at } \tau \gg \tau_{0}, \tau_{\text {rel }}
$$


The corresponding charm pair production rate per space-time unit reads

$\frac{D N_{c \bar{c}}}{d^{4} x}=\int d M^{2} \frac{d^{3} p_{1}}{(2 \pi)^{3}} \frac{d^{3} p_{1}}{(2 \pi)^{3}} f\left(p_{1}, x\right) f\left(p_{2}, x\right) \sigma\left(M^{2}\right) v_{r e l} \delta\left(\left[p_{1}+p_{2}\right]^{2}-M^{2}\right)$

$\left(v_{r e l}=\right.$ relative velocity of fusing partons). For a boost invariant distribution in the rapidity interval $d Y$ the yield, according to lowest order $g g \rightarrow c \bar{c}$ and $q \bar{q} \rightarrow c \bar{c}$ processes, for pairs with invariant mass $M$ and transverse momentum $q_{\perp}$ can be cast in the form

$\frac{d N_{c \bar{c}}^{b}}{d M^{2} d q_{\perp}^{2} d Y}=M^{2} \frac{N_{b} \sigma_{b}(M) R^{2}}{2^{7} \pi^{4}} \int_{\tau_{0}}^{\tau_{f}} d \tau \tau\left[J_{1}^{b}+2 J_{2}^{b}+J_{3}^{b}\right]$

In case of $b=q \ddot{q}$ one has

$$
\begin{aligned}
& \sigma_{q \bar{q}}=\frac{8 \pi \alpha_{s}^{2}}{27 M^{2}}\left(1+2 \frac{m_{c}^{2}}{M^{2}}\right) W\left(\frac{m_{c}}{M}\right), \quad N_{q \bar{q}}=72, m_{c}=1.5 \mathrm{GeV}, \\
& J_{1}^{q \bar{q}}=\left(\frac{\tau_{0}}{\tau} N_{0}^{q \bar{q}} \exp \left\{\frac{\tau_{0}-\tau}{\tau_{r e l}}\right\}\right)^{2} \frac{L}{M M_{\perp}}, N_{0}^{q \bar{q}}=\left(1-x_{0}\right) n_{0} p_{\perp}^{* l-3}(2 \pi)^{2}(l-3) g_{q \bar{q}}^{-1}, \\
& L= \begin{cases}\int_{p_{\perp}^{*}\left(M_{\perp}-p_{\perp}^{*}\right)}^{M^{2} / 4}\left(x\left(\frac{1}{4} M_{\perp}^{2}-x\right)^{-1 / 2}\left(x-\frac{1}{4} M^{2}\right)^{-1 / 2} x^{1-l}\right. & \text { for } M_{\perp}>p_{\perp}^{*}+\frac{M^{2}}{4 p_{\perp}^{*}} \\
\sum_{k=0}^{l-2}\left(\begin{array}{c}
l-2 \\
k
\end{array}\right) B\left(k+\frac{1}{2}, l-k-\frac{3}{2}\right)\left(\frac{M_{\perp}}{2}\right)^{-2 k-1}\left(\frac{M}{2}\right)^{2(k-l)+3} & \text { for } M_{\perp} \leq p_{\perp}^{*}+\frac{M^{2}}{4 p_{\perp}^{*}} .\end{cases}
\end{aligned}
$$

The $J_{1}$ term describes fusion processes of partons stemming from the initial distribution $f_{0}$, while $J_{2,3}$ incorporate processes of initial distribution partons with already equilibrated partons (described by $\mathcal{F}$ ) or among the equilibrated ones. The corresponding expressions for $J_{2,3}$ are rather involved and not displayed here.

In Fig. 4 the rate for $b=q \bar{q}$ is displayed for $\tau_{r e l}>\tau_{0, f}$, i.e., an idealized situation where the minijet structure of the quark distribution persists some time before relaxing. This rate is compared with the thermal rate $[15,16]$

$$
\frac{d N_{c \tilde{c}}^{b}}{d M^{2} d Y}=\frac{\lambda_{b}^{2} N_{b} \sigma_{b}(M) \pi R^{2}}{2(2 \pi)^{4} M^{2}} 3\left(T_{0}^{3} \tau_{0}\right)^{2}\left[H\left(\frac{M}{T_{0}}\right)-H\left(\frac{M}{T_{f}}\right)\right]
$$

where $H(x)=K_{0}(x) x^{2}\left(x^{2}+8\right)+K_{1}(x) 4 x\left(x^{2}+4\right)$. For $b=g g$ one has

$\sigma_{g g}=\frac{2 \pi \alpha_{s}^{2}}{3 M^{2}} \sigma_{e q .(5)}\left(\frac{m_{c}}{M}\right), \quad N_{g g}=\frac{1}{2} g_{g}^{2}, \quad \lambda_{g g}=\frac{l-3}{l-4} \frac{x_{0} n_{0} p_{\perp}^{*}}{T_{0}^{4}} \frac{\pi^{2}}{3 g_{g}}$,

and $b=q \bar{q}$ follows analogously with $\lambda_{q \bar{q}}=\lambda_{g g}\left(1-x_{0}\right) g_{g} /\left(x_{0} g_{g \bar{q}}\right)$. Here we have normalized the parton energy density and particle number in such a way that $n_{t h}=n_{p . l .}=n, \quad e_{t h}=$ $e_{p . l .}=e$ and $n_{g}=x n, n_{q \bar{q}}=(1-x) n$. This enables us to compare the minijet rate (10) with the thermal rate (14), both ones having the same $n, e$, see dashed line in Fig. 4. As expected the minijet quark-fusion rate exceeds also partially the thermal gluon rate (dotted line). This points to an enhanced charm production by a not yet equilibrated quark distribution immersed in an already thermalized gluon bath, according to the hot glue scenario [5]. However, since the ratio of $q \bar{q} / g g$ rates is proportional to $(1-x)^{2} / x^{2}$ a suppression of the quark weight $1-x$, as discussed in the previous section, also strongly suppresses the $q \vec{q}$ source: $x_{0}=0.8$ (instead of $x_{e q}=0.4$ as in Fig. 4) causes a reduction by a factor 37 . Therefore, if the quark admixture is indeed so small, charm probes very cleanly the gluon distribution $f_{g}$, while the previously discussed photons probe $f_{g} f_{q+\bar{q}}$. 


\section{Thermalization/dileptons}

\subsection{Parton thermalization}

Assuming that an initial distribution of partons scattered into a small volume element at midrapidity evolves according to a kinetic theory of Boltzmann type eq. (1), then the parton distribution of secondaries evolves according to eq. (8) with $[13,14]$

$\mathcal{F}(\tau)=\int_{\tau_{0}}^{\tau} \frac{d \tau^{\prime}}{\tau_{r e l}\left(\tau^{\prime}\right)} \exp \left\{-\frac{p_{\perp}}{T\left(\tau^{\prime}\right)} \sqrt{1+\left(\frac{\tau}{\tau^{\prime}} \operatorname{sh} \xi\right)^{2}}\right\}$

In this form one employs a relaxation time approximation of the collision integral. All details of the cross sections are accumulated in the time dependent relaxation time $\tau_{\text {rel }}(\tau)$. Inelastic processes are included too, since the particle number is not conserved. The temperature parameter $T$ is determined by the evolution of the energy density $e=\int d \Gamma E f$, cf. $[13,17]$.

Since we are going to consider dileptons with invariant mass $M \approx 2-3 \mathrm{GeV}$ the cut-off $p_{\perp}^{*}$ in the minijet motivated initial distribution (7) and possible contributions of softer partons prevents a straightforward use of $f_{0}(7)$. In this situation we resort to an initial distribution $f_{0}$ which is motivated by the Schwinger mechanism of parton pair production

$$
f_{0}=N \delta(\xi) \exp \left\{-p_{\perp}^{2} / K\right\}
$$

with $K$ as parameter of average transverse parton momentum $\left\langle p_{\perp}\right\rangle=\sqrt{4 K / \pi}$ and $N$ as normalization.

\subsection{Dilepton yield}

Within such a framework the dilepton yield is similar to eq. (9). For lowest order $\alpha_{s}, \alpha$ $q \bar{q} \rightarrow \overline{l l}$ processes it becomes

$$
\frac{d N_{\bar{l}}}{d M^{2} d M_{\perp}^{2} d Y}=\frac{5 R^{2} \alpha^{2}}{72 \pi^{3}} \int_{\tau_{0}}^{\tau_{f}} d \tau \tau \int_{-\infty}^{\infty} d \eta \int_{-\infty}^{\infty} d \xi_{1} \int_{p_{-}}^{p_{+}} \frac{d p_{\perp 1} p_{\perp 1} f\left(p_{\perp 1}, \xi_{1}, \tau\right) \bar{f}\left(p_{\perp 2}, \xi_{2}, \tau\right)}{\sqrt{p_{\perp 1}^{2} q_{\perp}^{2}-\left(p_{\perp 1} M_{\perp} \operatorname{ch}\left(\eta-\xi_{1}\right)-\frac{1}{2} M^{2}\right)^{2}}}
$$

$\left(p_{\perp 2}=\sqrt{M_{\perp}^{2}-2 M_{\perp} p_{\perp 1} \operatorname{ch}\left(\eta-\xi_{1}\right)+p_{\perp 1}^{2}}, \operatorname{sh} \xi_{2}=\frac{1}{p_{\perp 2}}\left(M_{\perp} \operatorname{sh} \eta-p_{\perp 1} \operatorname{sh} \xi_{1}\right), p_{ \pm}=\right.$ $\frac{1}{2} M^{2}\left[M_{\perp} \operatorname{ch}\left(\eta-\xi_{1}\right) \mp q_{\perp}\right]^{-1}, R$ is the transverse radius of the radiating region) and has been analyzed in Ref. [14]. According to estimates in Refs. [14, 18, 19] the yield at $M>$ $2 \mathrm{GeV}$ is expected to exceed the Drell Yan background (which ultimately dominates at higher M) at RHIC and LHC energies. The time evolution of the yield is displayed in Fig. 5. According to eqs. $(8,16,18)$ the yield contains terms $f f \propto f_{0} f_{0}(a)$, and $f_{0} \mathcal{F}(b)$, and $\mathcal{F} \mathcal{F}(c)$. As seen in Fig. 5 for large relaxation times (taken for simplicity as constant) the $f_{0} f_{0}$ term dominates, while for very short relaxation times $\tau_{\text {rel }} \leq \tau_{0}$ also the thermalized partons $\propto \mathcal{F F}$ gives a substantial contribution. Adding all contributions we find that $75 \%$ of the total yield is produced within $1 / 3$ of the lifetime of secondary partons. Therefore, if the parton source dominates the total yield (including also the hadron sources), the dileptons seem to probe mainly the initial distribution of secondaries and carry little 


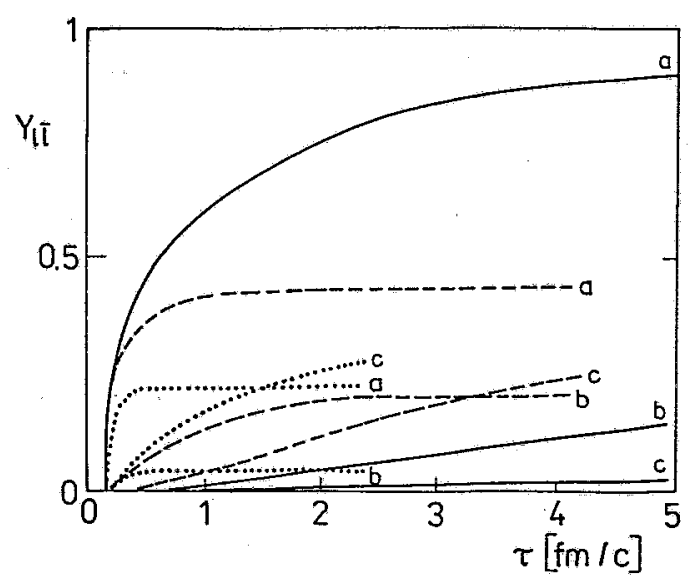

Figure 5. Time evolution of different contributions (see text) to the scaled dilepton yield $Y_{l \bar{l}}=\frac{d N_{l \bar{l}}}{d M^{2} d q_{\perp}^{2} d Y} 10^{3}$ $12 M^{2}(\hbar c)^{4} /\left(R^{2} \alpha^{2}\right)$ (in $\left.\mathrm{fm}^{2} \mathrm{GeV}^{2}\right)$ for pairs with $M=2 \mathrm{GeV}, q_{\perp}=0.2 \mathrm{GeV}$ (full/dashed/dotted lines: $\tau_{r e l}=10,1$, $0.13 \mathrm{fm} / \mathrm{c}) . \quad \tau_{0}=0.13 \mathrm{fm} / \mathrm{c}, K=1.2$ $\mathrm{GeV}^{2} . \tau_{f}$ is determined by the freezeout energy density corresponding to 200 $\mathrm{MeV}$.

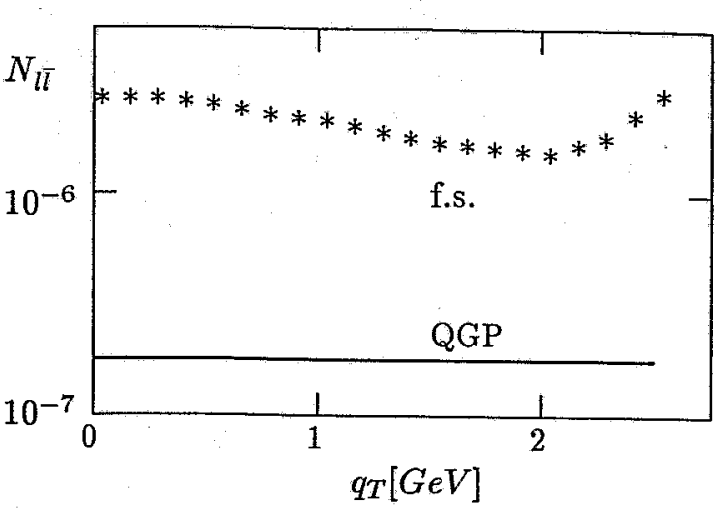

Figure 6. The dilepton spectrum eq. (18) $N_{l i} \equiv \frac{d N_{\bar{l}}}{d M_{\perp}^{2} d q_{\perp}^{2} d Y}$ (f.s., in $\mathrm{GeV}^{-4}$ ) as function of transverse momentum at $M_{\perp}=2.6$ $\mathrm{GeV}$. The charged hadron rapidity density is $d N_{c} / d y=10^{3}$, and $R=7 \mathrm{fm}, T_{f}=180 \mathrm{MeV}$, $K=1.2 \mathrm{GeV}^{2}, \tau_{0}=0.13$ (f.s.) and 1 (QGP) $\mathrm{fm} / \mathrm{c} . \tau_{f}$ is determined by the freeze-out energy density corresponding to $T_{f}$. QGP shows the ideal scaling of a longitudinally expanding massless quark-gluon plasma.

information from the later equilibrium (say quark-gluon plasma) era, unless the latter one lasts very long (e.g., in a long-living mixed phase).

Another interesting outcome of our investigations $[14,17]$ is that once a thermalized state is achieved the lateron evolution does not affect much the invariant mass spectrum of dileptons. E.g., in the HIJING code rather early the partons, due to trivial kinematics, pass through a state which looks to some extent thermal [6]. I.e., the dilepton spectrum is determined mainly by this state and the prior stages.

In case of $\tau_{r e l}>\tau_{0, f}$ the dominating $f_{0} f_{0}$ term results in

$$
\begin{aligned}
\frac{d N_{i \pi}}{d M_{\perp}^{2} d q_{\perp}^{2} d Y}= & \frac{5 \alpha^{2}}{9 \pi^{4} R^{2}}\left(\frac{d N_{c}}{d y} \frac{T_{f}}{K^{2}}\right)^{2} \log \left(\frac{d N_{c} / d y}{3 \pi R^{2} T_{f}^{3} \tau_{0}}\right) \exp \left\{-\frac{2 M_{\perp}^{2}+q_{\perp}^{2}}{4 K}\right\} \times \\
& \frac{\left(2 M_{\perp}^{2}-q_{\perp}^{2}\right) I_{0}\left(\frac{q_{\perp}^{2}}{4 K}\right)+q_{\perp}^{2} I_{1}\left(\frac{q_{\perp}^{2}}{4 K}\right)}{M_{\perp}\left(M_{\perp}^{2}-q_{\perp}^{2}\right)^{1 / 2}} .
\end{aligned}
$$

As seen in Fig. 6 (curve labeled by f. s.) this shows the so-called $M_{\perp}$ scaling: at $M_{\perp}=$ const the yield is fairly independent of $q_{\perp}$. We find that this also holds for the general case of a relaxing distribution $f(\tau)(8,16)[20]$. This generalizes the statement of Ref. [21] where both the Drell Yan contribution and the yield from a transversally expanding quark-gluon plasma are found to display approximate $M_{\perp}$ scaling. Any scale destroys the 
scaling, e.g., formfactors in the hadron gas cause a change of the yield by more than a factor 30 when going from $q_{\perp}=0$ to $2 \mathrm{GeV}$ at $M_{\perp}=2.6 \mathrm{GeV}$. Recent parton cascade results [22] display also strong violations of the $M_{\perp}$ scaling, however, it needs to be clarified whether this is a consequence (or artifact) of low- $p_{\perp}$ cut-offs and parton formfactors due to higher twist effects implemented in the code.

\section{Parton masses}

The above presented probes rely partially on undressed parton masses. To regularize certain cross sections we employ thermal masses $M^{*}(T) \propto g(T) T$. Such thermal masses might also be extracted from available lattice QCD data. SU(3) pure gauge theory data for a $16^{4}$ lattice [23] can be fitted by a family of thermodynamically self consistent equations of state [24] for interacting quasiparticles

$p=p_{t h}\left(T, M^{*}(T)\right)-p^{*}\left(M^{*}(T)\right), p_{t h}=\frac{g_{g}}{6 \pi^{2}} \int \frac{d k k^{4}}{\sqrt{k^{2}+M^{* 2}}}\left[\exp \left\{\frac{\sqrt{k^{2}+M^{* 2}}}{T}\right\}-1\right]^{-1}$.

Obvious choices are (i) $\partial p / \partial M^{*}=0$, which interrelates $M^{*}$ and $p^{*}[24,25]$, or (ii) $p^{*}=B$ = const, or (iii) $M^{*}=M=$ const. Such fits [26] yield (i) $M^{*} \propto T$ for $T / T_{c}=1.4-2.2$, or (ii) $M^{*}=0.5375 g(T) T, g^{2}=16 \pi^{2} /\left(11 \log \left(T / T_{c}\right)^{2}\right), B=0$ for $T / T_{c}=1.1-2.2$, or (iii) $p^{*} \propto T, M=0$. Besides the convenient parametrizations of the equation of state [27], which deviates strongly from the earlier popular bag model, the Ansätze (i, ii) point to substantial effective parton masses $m \approx \mathcal{O}(3) T_{c}$ in the available temperature range up to $2.2 T_{c}$ and indicate a divergent behaviour at $T_{c}$. Similar results are found in SU(2) gauge theory lattice data too [28].

These finite screening masses of course affect the various primordial plasma probes, as demonstrated in Ref. [25] for charm production. Screening masses in the off-equilibrium plasma represent a yet unsolved problem. Such masses might also be reponsible for destroying the $M_{\perp}$ scaling, which is conjectured to appear in any massless parton system.

\section{Summary}

In summary we present here analytical approaches to specific equilibration processes in evolving parton matter. As analog work [6] our presentation is aimed to analyze qualitatively some aspects which are throughoutly implemented in complex parton cascade codes. As pointed out by M. Gyulassy [29] the transition region of semihard (i.e., perturbatively tracktable) QCD proceses and softer ones represents a difficult region which needs more investigations with respect of reliable predictions of future RHIC and LHC results. 


\section{REFERENCES}

1. K. Geiger, B. Müller, Nucl. Phys. B369 (1992) 600,

K. Geiger, Phys. Rev. D46 (1992) 4965, 4986, D47 (1993) 133, NUC-MINN-93/5-T

2. X.N. Wang, M. Gyulassy, Phys. Rev. D45 (1992) 844

3. E.V. Shuryak, L. Xiong, Phys. Rev. Lett. 70 (1993) 2241

4. Proc. Quark Matter '91, Nucl. Phys. A544 (1992) 1c, (Eds.) T.C. Awes et al.

5. E.V. Shuryak, Phys. Rev. Lett. 68 (1992) 3270

6. T. Birò, E. van Dorn, B. Müller, M. Thoma, X.N. Wang, Duke-TH-93-46

7. B. Kämpfer, O.P. Pavlenko, FZR-93-09

8. T. Altherr, D. Seibert, CERN-TH.6882/93

9. E.V. Shuryak, talk at QM'93

10. J.I. Kapusta, P. Liechard, D. Seibert, Phys. Rev. D44 (1991) 2774

11. J. Alam, D.K. Srivastava, B. Sinha, D.N. Basu, Phys. Rev. D48 (1993)

12. K. Kajantie, P.V. Landshoff, J. Lindfors, Phys. Rev. Lett. 59 (1987) 2527

13. G. Baym, Phys. Lett. B138 (1984) 18

14. B. Kämpfer, O.P. Pavlenko, Phys. Lett. B289 (1992) 127

15. A. Shor, Phys. Lett. B215 (1988) 375

16. P. Koch, B. Müller, J. Rafelski, Phys. Rep. 142 (1986) 167

17. B. Kämpfer, O.P. Pavlenko, B. Heide, Proc. Int. Workshop Hirschegg XX (1992), (Ed.) H. Feldmeier, p. 245

18. K. Geiger. J.I. Kapusta, Phys. Rev. Lett. 70 (1993) 1920

19. J.I. Kapusta, L. McLerran, D.K. Srivastava, Phys. Lett. B283 (1992) 145

20. B. Kämpfer, O.P. Pavlenko, to be published

21. M. Asakawa, C.M. Ko, P. Lèvai, Phys. Rev. Lett. 70 (1993) 398

22. K. Geiger, NUC-MINN-93/10-T

23. J. Engels, J. Fingberg, F. Karsch, D. Miller, M. Weber, Phys. Lett. B252 (1990) 625

24. K.A. Bugaev, M.I. Gorenstein, B. Kämpfer, V.I. Zhdanov, Phys. Rev. D40 (1989) 2903

25. T. Birò, P. Lèvai, B. Müller, Phys. Rev. D42 (1990) 3078

26. B. Kämpfer, A. Peshier, to be published

27. D. Rischke, M.I. Gorenstein, A. Schäfer, H. Stöcker, W. Greiner, Phys. Lett. 278 (1992) 19

28. V. Goloviznin, H. Satz, poster T-43 presented at QM'93

29. M. Gyulassy, talk at QM'93 